\title{
Cachexia in Patients with Gastrointestinal Cancers: Contributing Factors, Prevention, and Current Management Approaches
}

My Editor's Pick for this issue is the excellent paper by Grundmann et al., which is focussed on cachexia's pathophysiology, emerging diagnostic criteria with potential biomarkers, prevention strategies, and

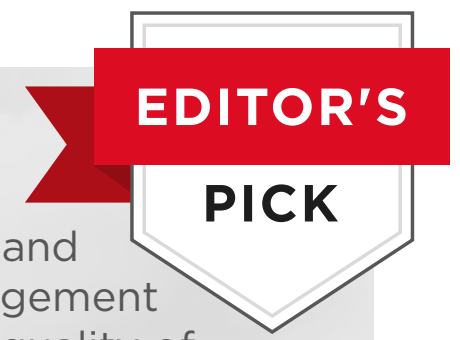
novel treatment approaches. We all hope for a more effective management and for a quicker amelioration of cachexia which negatively affects quality of life, responsiveness to chemotherapy, and survival in advanced cancer patients.

Authors:

*Oliver Grundmann, ${ }^{1,2}$ Saunjoo L. Yoon, ${ }^{2}$ Joseph J. Williams ${ }^{3}$

1. College of Pharmacy, Department of Medicinal Chemistry, University of Florida, Gainesville, Florida, USA

2. College of Nursing, Department of Biobehavioral Nursing Science, University of Florida, Gainesville, Florida, USA

3. Sunshine Integrative Health, Gainesville, Florida, USA

*Correspondence to grundman@ufl.edu

Disclosure: $\quad$ The authors have declared no conflicts of interest.

Acknowledgements: The figure was generated using BioRender.

Received: $\quad 21.07 .20$

Accepted: $\quad 07.09 .20$

Keywords: Cachexia, inflammation, presystemic drug metabolism, symbiosis.

Citation: $\quad$ EMJ Gastroenterol. 2020;9[1]:62-70.

\begin{abstract}
Cancer cachexia is highly prevalent among patients with the advanced stage of cancers and leads to a higher risk of mortality. Delayed management of cachexia results in suboptimal treatment outcomes and irreversible progression to refractory cachexia. The purpose of this review is to provide the pathophysiology of cancer cachexia, emerging diagnostic criteria with potential biomarkers, prevention strategies, and novel treatment approaches. Cachexia is characterised by the presence of an inflammatory process in conjunction with muscle mass and unintentional body weight loss. Various biomarkers such as leptin, ghrelin, TNFa, essential amino acids, total amino acids, and $C$-reactive protein are indicative of cachexia. Increased circulating levels of $\beta$-dystroglycan, myosin heavy-chain, and dystrophin are indicators of shortened survival time as skeletal muscle tissues break down. Despite muscle wasting being a hallmark of cachexia, recommended cachexia management is limited to nutritional counselling and administration of an appetite stimulant and corticosteroids for a short period, which often fail to reverse cancer cachexia. It is critical to monitor weight loss using the cachexia grading system for early detection, to halt progression to refractory cachexia and improve the survival of patients with cancer cachexia.
\end{abstract}




\section{INTRODUCTION}

Cancer cachexia is highly prevalent among patients with the advanced stages of cancers, affecting an estimated 12 million people worldwide and being causative in up to 2 million deaths annually as of 2016. ${ }^{1}$ A distinguishing feature of cachexia is the loss of musculoskeletal lean body mass, with or without fat mass loss, in conjunction with weight loss of $>5 \%$ over the course of 6 months. ${ }^{2}$ These metabolic derangements delineate it from age-related sarcopenia or malnutrition, which can be reversed with proper nutritional supplementation or exercise. ${ }^{3}$

Cancer-associated cachexia is often linked to increased morbidity and mortality given its underdiagnosis and delayed treatment. ${ }^{4,5}$ Delayed diagnosis of cachexia decreases quality of life, and may delay optimal patient care if systemic inflammation and gastrointestinal (GI) symptoms hinder the administration of necessary chemotherapy. ${ }^{6}$ Approximately $80 \%$ of patients with advanced cancers experience cachexia, at which point intervention measures are often too late to reverse the condition and the progressive nature of the malignancy is accelerated by the complex cachectic metabolic derangement. Patients with advanced GI cancers may present with a higher incidence of cachexia given the aggressive nature of chemotherapy, the nutritional deficiency caused by the malignancy, and the relative proximity of localised inflammation and systemic responses. ${ }^{7}$ This review addresses the pathophysiology of cancer cachexia, emerging diagnostic criteria and potential biomarkers, prevention strategies, and current as well as novel treatment approaches that either slow, halt, or reverse the progression of cachexia in patients with Gl cancer.

\section{PATHOPHYSIOLOGY OF CANCER CACHEXIA}

The multifactorial nature of cancer development itself is highly complex, so it comes as no surprise that the pathophysiology of cachexia within this specific setting remains poorly understood. However, cachexia can be clearly differentiated from both malnutrition and sarcopenia by the presence of an inflammatory process in conjunction with muscle mass and body weight loss. ${ }^{3}$ The systemic metabolic derangement caused by the malignancy is a result of hypercatabolism, hypermetabolism, systemic inflammation, and an imbalance in protein synthesis regulation. ${ }^{8,9}$ Regulation of caloric intake is mediated through a variety of hormones, among them the adipocyte-generated cytokine-associated hormone leptin, the orexigenic peptide ghrelin present primarily in the GI tract, and the neuropeptide a-melanocytestimulating hormone. ${ }^{10-12}$ Under physiological conditions, upon food intake leptin is released into the blood circulation to stimulate the production of proopiomelanocortin, leading to the release of cortisol and ultimately suppression of further food consumption (Figure 1). ${ }^{13}$ Its opposing hormone, ghrelin, stimulates appetite by increasing the release of neuropeptide $Y$ and orexin in the central nervous system, leading to increased gastric acid secretion (Figure 1).13,14 A higher amount of leptin is expressed in patients with GI cancers, while a lower amount of ghrelin is present in this population, contributing to a deranged metabolism and lower caloric intake. ${ }^{15}$ The imbalance between leptin and ghrelin is primarily attributed to systemic inflammation, an early hallmark of cancer and a necessary contributor to the development of cachexia. In fact, weight loss of $<5 \%$ over 6 months but increasing inflammatory markers may indicate a precachectic state that warrants intervention to prevent progression. ${ }^{16,17}$ It has also been observed that patients with cachexia develop resistance to ghrelin even if the hormone is being supplemented to stimulate appetite. ${ }^{18}$

A nonspecific marker of systemic inflammation is C-reactive protein (CRP), which increases in the early stages of malignancy. More specific to GI cancers are elevated plasma levels of TNFa, $\mathrm{IL}-1 \mathrm{~B}$, and IL-6, as well as a reduction in serum albumin and adiponectin levels. ${ }^{15}$ While TNFa is not a specific marker of cancer cachexia, its increased blood levels in conjunction with rising IL-6 levels correlate with the progression from malnutrition to cachexia (Figure 1). ${ }^{15}$ Interestingly, expression levels of IL-1 $\beta$ were found to be elevated in adipose and tumour tissues of $\mathrm{Gl}$ cancer patients with cachexia versus those without cachexia, indicating more pronounced crosstalk between the tumour and surrounding tissues as a contributing factor in the development of cachexia. ${ }^{19}$ Along with higher 
IL-1及 expression in patients with cachexia comes an increase in fibrosis and macrophage infiltration in subcutaneous adipose tissue compared to weight-stable patients with cancer, suggesting both an inflammatory and morphological differentiation in $\mathrm{Gl}$ cancer cachexia. ${ }^{20}$

Because of a shift in metabolic and catabolic activity, both lipid and protein, as well as musclerelated biomarkers, may indicate sarcopenia and malnutrition. Increased local and systemic inflammation due to the tumour cause muscle proteolysis, in conjunction with malabsorption of nutrients due to the localised presence of the malignancy. The ratio of essential amino acids to total amino acids and CRP in plasma was higher in patients with $\mathrm{Gl}$ cancer who lost psoas muscle area compared to those who maintained or gained psoas muscle. ${ }^{21}$ This association between inflammation, increased proteolysis, and loss of muscle mass indicates that patients with advanced GI cancer have deranged metabolic/ catabolic activity that cannot be compensated with nutritional supplementation alone. Other indicators of loss of muscle mass are increased plasma levels of $\beta$-dystroglycan, myosin heavychain, and dystrophin, which play a vital role in providing structural integrity to muscle tissues. ${ }^{22}$ Increased circulating levels are indicators of shortened survival time and refractory cachexia as skeletal muscle tissues are breaking down.

\section{EMERGING DIAGNOSTIC CRITERIA AND BIOMARKERS}

Patients with $\mathrm{Gl}$ cancer remain at high risk of developing cancer cachexia and a majority are diagnosed too late for effective prevention or treatment to slow or reverse the progression of muscle and weight loss.

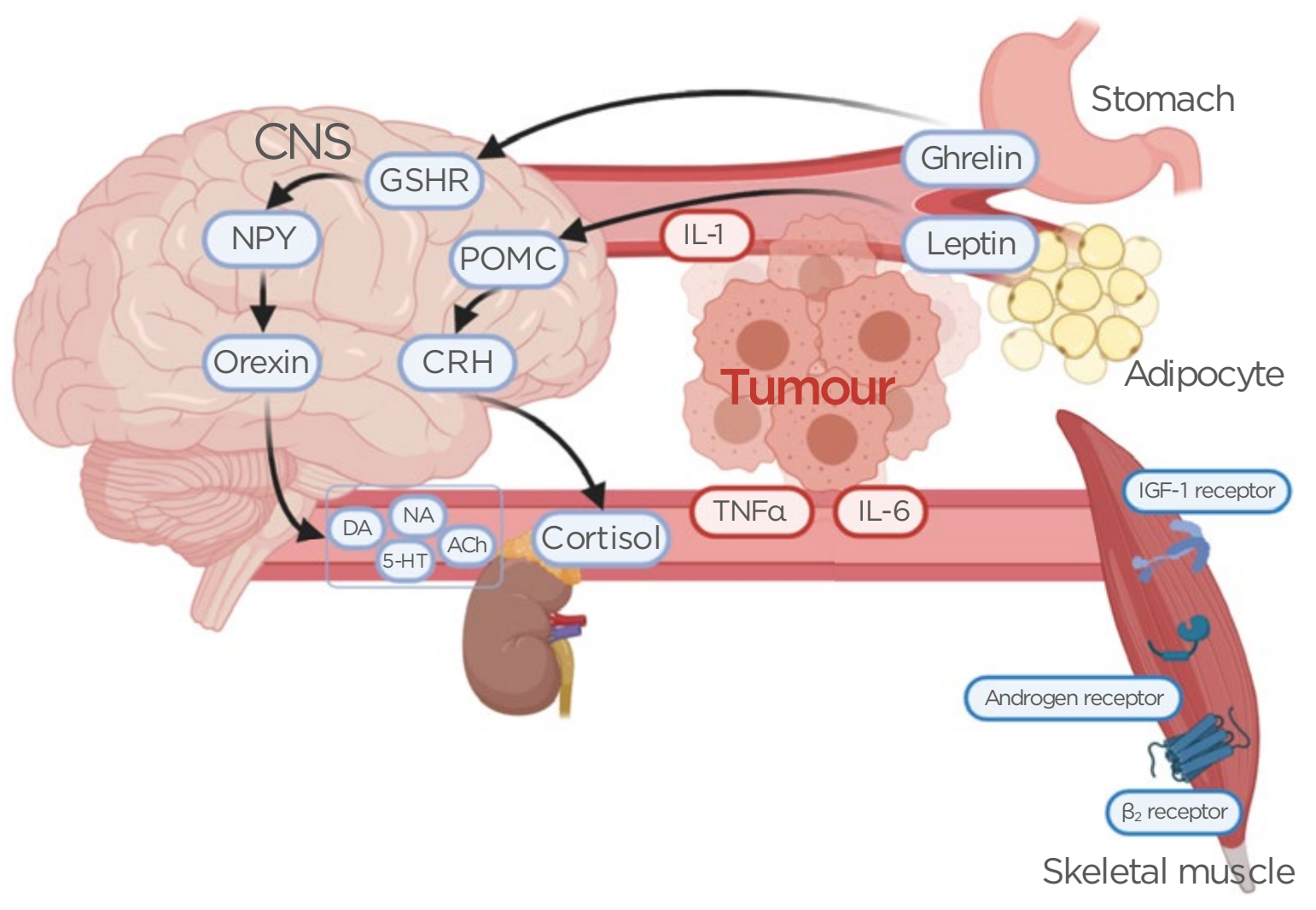

Figure 1: Effects of ghrelin and leptin secretion on the release of hormones and neurotransmitters from the central nervous system.

Release of inflammatory mediators from the tumour alters ghrelin and leptin homeostasis, leading to reduced skeletal muscle tissue. Also shown are potential targets for pharmacotherapeutic intervention, such as GSHR, IGF-1 receptor agonists, androgen receptor agonists, and adrenergic $\beta_{2}$ receptor antagonists.

ACh: acetylcholine; CNS: central nervous system; CRH: corticotropin-releasing hormone; DA: dopamine; GSHR: ghrelin receptor agonists; IGF-1: insulin-like growth factor-1; NA: noradrenaline; NPY: neuropeptide Y; POMC: proopiomelanocortin; 5-HT: serotonin. 
Delayed diagnosis leads to increased morbidity and mortality, lower quality of life, and suboptimal therapeutic outcomes. ${ }^{4,5}$ Early and frequent evaluation of patients with $\mathrm{Gl}$ cancer is of critical value to detect weight loss as well as early changes in clinical and metabolic status. Such changes fall in the category of precachexia if inflammatory and/or nutritional markers are changing, and nutritional needs evolve either independently or based on chemotherapy or radiation treatment (Table 1). ${ }^{23}$ The most common diagnostic criteria are nutritional assessments, weight loss $>5 \%$ over 6 months without starvation, $>2 \%$ of weight loss if BMI $<20 \mathrm{~kg} / \mathrm{m}^{2}$, and/or demonstration of sarcopenia via skeletal muscle index measurement. ${ }^{2}$

Nutritional assessment tools are often used in conjunction with weight changes and quality of life observations in diagnosed patients. Among the established scales, the Nutrition Risk Screening-2002 (NRS-2002), Malnutrition
Universal Screening Tool (MUST), and the Malnutrition Screening Tool (MST) are frequently used in clinical practice to establish nutritional and metabolic derangements. ${ }^{16}$ If a patient has been identified to be at risk for developing cachexia or is precachectic, a more in-depth evaluation of nutritional intake, physical activity, and body composition has to be considered, along with nutrition assessment tools, such as the Subjective Global Assessment (SGA) or Minimal Nutrition Assessment (MNA), to evaluate the degree of malnutrition and existing cachexia. ${ }^{16,24}$ The combined use of physical diagnostic criteria and nutritional screening or assessment tools has been given strong recommendations by the European Society for Clinical Nutrition and Metabolism (ESPEN) despite a "very low" level of available evidence.16,25 This has been further refined as a two-step model for risk screening and diagnosis assessment of malnutrition by the Global Leadership Initiative on Malnutrition (GLIM), first convened in 2016. ${ }^{26}$

Table 1: Stages and characteristics of cachexia.

\begin{tabular}{|c|c|}
\hline \multirow{3}{*}{$\begin{array}{l}\text { Precachexia } \\
\text { Weight loss }<5 \% \\
\text { Clinical and metabolic changes }\end{array}$} & Monitor weight and manage nutritional needs \\
\hline & $\begin{array}{l}\text { Frequent evaluation of inflammatory markers (TNFa, IL-1, IL-6) and nutrition } \\
\text { markers (ghrelin and leptin) }\end{array}$ \\
\hline & Consider changes in chemotherapy or radiation regimen to prevent progression \\
\hline \multirow{5}{*}{$\begin{array}{l}\text { Cachexia } \\
\text { Weight loss }>5 \% \\
\text { Different phenotypes }\end{array}$} & Evaluate organ function, especially liver and kidney \\
\hline & Counsel on diet and exercise, suggest additional protein intake per European \\
\hline & Society for Clinical Nutrition and Metabolism (ESPEN) recommendations \\
\hline & Pharmacotherapy intervention to increase appetite and anabolism \\
\hline & Counselling on weight maintenance or recovery, depending on cancer stage \\
\hline \multirow{4}{*}{$\begin{array}{l}\text { Refractory Cachexia } \\
\text { Refractory catabolic cancer disease }\end{array}$} & Palliative care to reduce pain and maintain the level of quality of life \\
\hline & Associated with poor outcomes and high mortality in patients with cancer \\
\hline & Pharmacotherapeutic options can be exhausted according to patient well-being \\
\hline & Consider parenteral nutrition support and maintenance of hydration \\
\hline
\end{tabular}


The latest GLIM criteria as of 2019 include unintentional weight loss, low BMI, and reduced muscle mass as phenotypic criteria, and reduced food intake and inflammation or disease burden as aetiologic criteria, of which at least one phenotypic and aetiologic criterion need to be present to diagnose malnutrition. ${ }^{26}$

Patients with a weight-stable condition and BMI $\geq 25 \mathrm{~kg} / \mathrm{m}^{2}$ demonstrated longer survival than the patients who lost weight. ${ }^{27}$ While cachexia is characterised by lean muscle loss, the association of BMI by \% of weight loss can predict the prognosis of patients with cachexia, quality of life, and symptom burden. The cachexia grading system (ranged 0-4), based on \% of weight loss and BMI, is beneficial for early detection to manage cancer cachexia. ${ }^{27,28}$

Thus, the ideal goal would be to prevent the development of cancer cachexia in the first place by recognising potential cachexia in patients with $\mathrm{Gl}$ cancers. Once cachexia has progressed past a particular point, muscle degradation and loss of physical functioning are irreversible and impact the success of therapy and outcome. Patients with $\mathrm{Gl}$ cancers are at higher risk of death if they had developed cachexia or refractory cachexia, lower grades in phase angle, decreased handgrip strength, and an increased CRP. ${ }^{29}$ Together with weight loss, these measures can be utilised to evaluate the progression of muscle strength loss and increased inflammation to provide potential intervention. The phase angle, a composite measure obtained by bioelectrical impedance analysis, can predict nutritional status and overall health status. ${ }^{30}$

Another early marker of cachexia in Gl cancer is carnosine dipeptidase 1, which has been associated with weight loss, malnutrition, lipid breakdown, and low circulating albumin as well as insulin-like growth factor $1 .{ }^{31}$ The plasma levels of the enzyme, which plays a role in several disease states and is primarily expressed in the central nervous system and the liver, is significantly reduced in patients who develop cachexia compared to weight-stable patients with $\mathrm{Gl}$ cancer. Carnosine is highly concentrated in muscle tissue, serving as a $\mathrm{pH}$ buffer to balance aerobic and anaerobic metabolism and catabolism activity. ${ }^{32}$ Because muscle integrity and degradation is a contributing factor to cachexia, elevated plasma levels of $\beta$-dystroglycan can serve as specific biomarkers for the diagnosis of $\mathrm{Gl}$ cancer cachexia, while elevations in dystrophin and myosin heavy-chain may predict poor survival..22 Another potential predictor of muscle degradation in patients with Gl cancer with cachexia are serum levels of carnitine, an essential compound needed in fatty acid energy metabolism in skeletal muscle cells. ${ }^{33}$ Carnitine serum levels were significantly lower in patients with Gl cancer with cachexia compared to other patients with cachexia and healthy controls, potentially providing a specific marker for the severity of cachexia in patients with cancer.

Despite these emerging biomarkers for cachexia development and progression, none are routinely used in clinical practice or have been tested widely as screening tools.

Current clinical practice guidelines for cachexia diagnosis primarily rely on the overall patient status by evaluating subjective symptoms, taking a history, a clinical examination, body composition measures, general laboratory values, and activity monitoring (Table 2). ${ }^{16,34}$ While this approach can identify cachexia, it is often not specific or sensitive enough to monitor the development of progression in a timely manner for providing appropriate intervention. Clinicians may, therefore, consider additional laboratory measures as discussed above to guide important pharmacologic and nonpharmacologic treatment decisions to prevent or halt the progression of cancer cachexia before it advances to the mostly treatment-resistant refractory cachexia stage (Table 1).

\section{PREVENTION STRATEGIES FOR GASTROINTESTINAL CANCER CACHEXIA}

Cancer cachexia contributes to an increased risk of premature death in patients with Gl cancer; hence, preventing its development remains a primary challenge and opportunity to improve quality of life and patient outcomes. Given that sudden and unexpected weight loss is both a hallmark indicator for tumour growth and anorexia-cachexia, it may serve as a nonspecific but leading sign for clinicians to investigate further. Any patient diagnosed with cancer is at risk of developing cachexia and therefore should 
be frequently monitored for weight loss, changes in appetite and caloric intake, decrease in muscle strength, and increased inflammation.

Given the profound loss of lean muscle mass and metabolic derangement, nutritional intervention serves as an initial and ongoing therapeutic intervention to prevent or potentially halt or reverse the progression of cancer cachexia. Specifically, protein intake should be increased to at least $1 \mathrm{~g} / \mathrm{kg} /$ day, ideally to $1.5 \mathrm{~g} / \mathrm{kg} /$ day, in combination with regular physical activity or exercise. ${ }^{16}$ Regular physical activity or exercise in conjunction with adequate nutrition is essential to maintain muscle strength, physical functioning, and metabolic activity. ${ }^{35}$

Rising serum levels of CRP, TNFa, and IL-6, in conjunction with $>5 \%$ weight loss over 6 months and decreased muscle strength, is a strong indicator for a cachexia diagnosis. ${ }^{36}$ Hence, a prevention strategy that is commonly employed in patients with cancer has been physical exercise to maintain muscle strength and nutritional support for caloric intake. ${ }^{37,38}$ Physical exercise has been studied in several clinical trials for the prevention and treatment of cachexia in patients with cancer, and evaluated in a systematic Cochrane review. ${ }^{39}$ Despite agreement among clinicians and researchers that exercise does benefit patients with precachexia and cachexia, heterogeneity in study design and neglect to include cachexia staging and assessment prevent consistent evaluation of the safety and efficacy of exercise in cachexia. Hence, its benefit remains undetermined and clinicians are left to consider its recommendation on an individual patient basis. Similar to physical exercise, nutritional support is a commonly employed and clinically utilised adjunct therapy to prevent and treat anorexia, malnutrition, and cachexia.

However, enteral nutrition support with omega-3 fatty acids, arginine, glutamine, and polyribonucleotides has not shown consistent improvements or increased survival in patients with Gl cancer, and may only benefit patients with good functional status and an overall better prognosis. ${ }^{40,41}$ Systemic inflammation remains a major contributing factor in the development of cancer cachexia and also serves as a biomarker for its diagnosis as previously discussed. Both the tumour and the immune response contribute to the development of a precachectic state that leads to a metabolic instability, hastening weight and muscle loss. ${ }^{42}$

Table 2: Current guidelines to diagnose cancer-associated cachexia.

\begin{tabular}{|l|r|}
\hline Subjective symptoms & $\begin{array}{r}\text { Appetite, early satiety, nausea, vomiting, disturbances of taste or smell, } \\
\text { other Gl symptoms, weakness, disease-related burden, quality of life }\end{array}$ \\
\hline History & Weight change, speed of weight loss, \% of normal dietary intake \\
\hline Clinical examination & $\begin{array}{r}\text { Inspection of mouth, abdomen, hydration status, oedema, body weight, } \\
\text { perceived physical strength }\end{array}$ \\
\hline Laboratory values & $\begin{array}{r}\text { Performance status (ECOG or Karnofsky Performance Scale), upper limb } \\
\text { hand-grip dynamometry, body-worn activity meters }\end{array}$ \\
\hline Activity monitoring & $\begin{array}{r}\text { Cross-sectional imaging (CT or MRI), dual energy X-ray imaging (DEXA), } \\
\text { anthropometry (mid-arm muscle area), bioelectrical impedance analysis }\end{array}$ \\
\hline Body composition &
\end{tabular}

CRP: C-reactive protein; DEXA: dual-energy X-ray absorptiometry; ECOG: Eastern Cooperative Oncology Group; GI: gastrointestinal.

Adapted from Radbruch et al. ${ }^{34}$ 
Reducing or suppressing systemic inflammation can potentially reduce both the progression of the malignancy as well as the development of a cachectic state. The use of anticytokine drugs, such as thalidomide, that target a range of proinflammatory cytokines (TNFa, IL-6, IL$1 \beta$, etc.) has shown mixed results in treating or preventing cachexia in clinical trials to date, primarily due to a heterogeneous patient population and testing in late disease states. ${ }^{43}$ There is a potential correlation between the use of anti-inflammatory drugs, in particular the long-term use of aspirin, and lowering the risk of colorectal cancer development, as has been shown in several longitudinal studies. ${ }^{44,45}$

\section{CURRENT AND NOVEL APPROACHES TO CANCER CACHEXIA TREATMENT}

The two primary goals in the prevention and treatment of cancer cachexia are to increase or maintain appetite and to prevent a loss of muscle mass. Although weight and appetite loss are associated, they are not always an indication of cachexia since chemotherapy and radiation therapy themselves can impact appetite and weight changes through systemic and local inflammation themselves. ${ }^{46}$ Because the imbalance in catabolism and anabolism is caused by systemic inflammation, the most common first-line pharmacotherapies remain glucocorticoids and progesterone derivatives that aim to stimulate appetite and maintain or increase weight via anabolism. ${ }^{47,48}$ However, both drug classes have limited long-term benefits and do not improve physical functioning. Megestrol acetate remains the primary agent used to prevent and treat all stages of cancer cachexia and clinical studies indicate weight stabilisation or weight gain with its short-term use. ${ }^{49}$ Combination therapy with nonsteroidal anti-inflammatory agents to suppress inflammation did not show a benefit over treatment with megestrol acetate alone, and thus remains limited to use in clinical trials. $^{50,51}$ Other agents that are occasionally used with mixed or equivocal success are cannabinoids, anticytokine and anabolic agents, and $\beta$-blockers (Figure 1). ${ }^{23}$ Each class of agents has limitations and none of the experimental off-label uses has shown consistent benefits in halting or reversing cachexia in patients with Gl cancer specifically or patients with advanced cancer in general. Such is the case with the combined use of agents that stimulate protein synthesis, such as short-term use of glucocorticoids or omega-3 fatty acids, ${ }^{52}$ and agents that prevent catabolism, specifically thalidomide, which downregulates the ubiquitinproteasome proteolysis pathway involved in protein degradation. ${ }^{48}$ Thalidomide remains controversial due to its known genotoxic and other adverse effects and has not been approved for the prevention or treatment of cancer cachexia. Its benefit in this population is also questionable given limited evidence from clinical studies. ${ }^{53}$ Newer drugs that are being investigated target specific signalling pathways involved with food intake. Among them is the ghrelin receptor agonist anamorelin that has shown some benefits in patients with non-small cell lung carcinoma (ROMANA1 and ROMANA2 studies, including a total of 979 patients) $)^{54,55}$ and one multicentre study in 50 patients with Gl cancer..$^{56}$ Anamorelin does modestly improve body weight and lean body mass over the course of 12 weeks in patients with cancer cachexia compared to those receiving placebo; however, the European Medicines Agency (EMA) did not grant market approval for this indication in non-small cell lung cancer. Aside from increased strength exercises, physical functioning remains unaddressed in clinical trials to date.

Pharmacological approaches have limitations due to side effects and added burden to patients with cachexia, who are taking multiple medications and experiencing treatment-associated side effects. Several nonpharmacological approaches are utilised to improve the quality of life and mitigate the limitations of current pharmacotherapy to overcome the adverse effects of cancer chemotherapy. Such approaches involve targeted acupuncture to reduce specific $\mathrm{Gl}$ and cachexia symptoms, ${ }^{57}$ nutritional counselling, psychosocial interventions, and dietary supplements. ${ }^{58}$ Recent guidelines recommended using enteral tube feeding and parenteral nutrition only with caution, and not treating patients with these approaches consistently. ${ }^{59}$

\section{CONCLUSION}

Cancer-associated cachexia has received increased attention for the last two decades. The definition of cachexia has been generally agreed 
upon in the cachexia research community; however, diagnostic measures using the biological markers are still mainly under investigation. Recently, evaluation of the skeletal muscle index and psoas muscle index is often used to assess the loss of muscle mass for measuring cachexia. Despite various pharmacological agents having undergone clinical trials and some shown promising results, currently no medications are available to treat cachexia. Most recent guidelines for cancer cachexia management recommend dietary counselling, megestrol acetate as an appetite stimulant, or short-term use of dexamethasone. ${ }^{58}$ These medications may be helpful to stimulate appetite but should not be taken for a long time due to various side effects. Also, these medications or nutritional counselling may not slow down lean muscle loss or treat cachexia. If possible, nonpharmacological approaches that would not be burdensome for patients may be a promising solution for patients with cachexia, who are affected by fatigue, decreased energy levels, nausea, and decreased appetite.

\section{References}

1. von Haehling $S$ et al. Prevalence and clinical impact of cachexia in chronic illness in Europe, USA, and Japan: facts and numbers update 2016. J Cachexia Sarcopenia Muscle. 2016;7(5):507-9.

2. Fearon $\mathrm{K}$, et al. Definition and classification of cancer cachexia: an international consensus. Lancet Oncol. 2011:12(5):489-95.

3. Evans WJ et al. Cachexia: a new definition. Clin Nutr. 2008;27(6) .793-9.

4. Buskermolen S et al. Weight loss of $5 \%$ or more predicts loss of fat-free mass during palliative chemotherapy in patients with advanced cancer: a pilot study. Nutr Cancer. 2012;64(6):826-32.

5. Zhou T et al. Development and validation of a clinically applicable score to classify cachexia stages in advanced cancer patients. J Cachexia Sarcopenia Muscle. 2018;9(2):306-14.

6. Baracos VE et al. Cancer-associated cachexia. Nat Rev Dis Primers. 2018;4:17105.

7. Yoon SL et al. Predicting unintentional weight loss in patients with gastrointestinal cancer. J Cachexia Sarcopenia Muscle. 2019;10(3):526-35

8. Riccardi DMDR et al. Plasma lipid profile and systemic inflammation in patients with cancer cachexia. Front Nutr. 2020;7:4.

9. Peixoto da Silva S et al. Cancer cachexia and its pathophysiology: links with sarcopenia, anorexia and asthenia. J Cachexia Sarcopenia Muscle. 2020;11(3):619-35.

10. Timper K, Brüning JC. Hypothalamic circuits regulating appetite and energy homeostasis: pathways to obesity. Dis Model Mech. 2017;10(6):679-89

11. Zanchi D et al. The impact of gut hormones on the neural circuit of appetite and satiety: a systematic review. Neurosci Biobehav Rev. 2017;80:457-75.

12. Chaudhri $O$ et al. Gastrointestinal hormones regulating appetite. Philos Trans R Soc Lond Biol Sci. 2006;361(1471):1187-209.

13. Kerem $\mathrm{M}$ et al. Adipokines and ghrelin in gastric cancer cachexia. World J Gastroenterol. 2008;14(23):3633-41.

14. DeBoer MD. Ghrelin and cachexia: will treatment with GHSR-1a agonists make a difference for patients suffering from chronic wasting syndromes? Mol Cell Endocrinol. 2011;340(1):97-105.

15. Kemik $O$ et al. The relationship among acute-phase response proteins, cytokines, and hormones in various gastrointestinal cancer types patients with cachectic. Hum Exp Toxicol. 2012;31(2):117-25

16. Arends $\mathrm{J}$ et al. ESPEN guidelines on nutrition in cancer patients. Clin Nutr. 2017;36(1):11-48.

17. Laviano A et al. Cachexia: clinical features when inflammation drives malnutrition. Proc Nutr Soc. 2015;74(4):348-54.

18. Fujitsuka $\mathrm{N}$ et al. Potentiation of ghrelin signaling attenuates cancer anorexia-cachexia and prolongs survival. Transl Psychiatry. 2011;1(7):e23.

19. de Matos-Neto EM et al. Systemic inflammation in cachexia - is tumor cytokine expression profile the culprit? Front Immunol. 2015;6:629.

20. Batista ML et al. Cachexia-associated adipose tissue morphological rearrangement in gastrointestinal cancer patients. J Cachexia Sarcopenia Muscle. 2016;7(1):37-47.

21. Kitagawa $M$ et al. High serum essential amino acids as a predictor of skeletal muscle depletion in patients with cachexia and advanced gastrointestinal cancers. Nutr Clin Pract. 2017;32(5):645-51.
22. Stephens NA et al. Evaluating potential biomarkers of cachexia and survival in skeletal muscle of upper gastrointestinal cancer patients. J Cachexia Sarcopenia Muscle. 2015;6(1):53-61.

23. Grundmann $O$ et al. "Malnutrition, Cachexia and Quality of Life in Patients with Cancer," Preedy VR, Patel VB (eds), Handbook of Famine, Starvation, and Nutrient Deprivation: From Biology to Policy (2019), Cham: Springer International Publishing, pp.1-18.

24. Isenring $\mathrm{E}$ et al. Nutritional status and information needs of medical oncology patients receiving treatment at an Australian public hospital. Nutr Cancer. 2010;62(2) :220-8.

25. Castillo-Martínez $L$ et al. Nutritional assessment tools for the identification of malnutrition and nutritional risk associated with cancer treatment. Rev Invest Clin. 2018;70(3):121-5.

26. Jensen GL et al. GLIM criteria for the diagnosis of malnutrition: a consensus report from the global clinical nutrition community. JPEN J Parenter Enteral Nutr. 2019;43(1) :32-40.

27. Martin L et al. Cancer cachexia in the age of obesity: skeletal muscle depletion is a powerful prognostic factor, independent of body mass index. J Clin Oncol. 2013;31(12) :1539-47.

28. Daly $L$ et al. The relationship between the BMI-adjusted weight loss grading system and quality of life in patients with incurable cancer. J Cachexia Sarcopenia Muscle. 2020;11(1):160-8.

29. Ozorio GA et al. Cachexia stage, patient-generated subjective global assessment, phase angle, and handgrip strength in patients with gastrointestinal cancer. Nutr Cancer. 2017;69(5):772-9.

30. Grundmann $\mathrm{O}$ et al. The value of bioelectrical impedance analysis 
and phase angle in the evaluation of malnutrition and quality of life in cancer patients--a comprehensive review. Eur J Clin Nutr. 2015;69(12):1290-7.

31. Arner $\mathrm{P}$ et al. Circulating carnosine dipeptidase 1 associates with weight loss and poor prognosis in gastrointestinal cancer. PLoS One. 2015:10(4):e0123566.

32. Smith EC. The buffering of muscle in rigor; protein, phosphate and carnosine. J Physiol. 1938;92(3):33643.

33. Malaguarnera $M$ et al. Decrease of serum carnitine levels in patients with or without gastrointestinal cancer cachexia. World J Gastroenterol. 2006:12(28):4541-5.

34. Radbruch $L$ et al. Clinical practice guidelines on cancer cachexia in advanced cancer patients. Department of Palliative Medicine/ European Palliative Care Research Collaborative; Cancer Cachexia Hub. 2010. Available at: http://www. cancercachexia.com/literaturewatch/43_clinical-practiceguidelines-on-cancer-cachexia-inadvanced-cancer. Last accessed: 1 September 2020.

35. Glover El, Phillips SM. Resistance exercise and appropriate nutrition to counteract muscle wasting and promote muscle hypertrophy. Curr Opin Clin Nutr Metab Care. 2010;13(6):630-4.

36. Costa RGF et al. Cancer cachexia induces morphological and inflammatory changes in the intestinal mucosa. J Cachexia Sarcopenia Muscle. 2019;10(5):1116-27.

37. Muscaritoli M et al. Prevention and treatment of cancer cachexia: new insights into an old problem. Eur J Cancer. 2006;42(1):31-41.

38. Garla P et al. Nutritional therapy in gastrointestinal cancers. Gastroenterol Clin North Am. 2018;47(1):231-42

39. Grande AJ et al. Exercise for cancer cachexia in adults: executive summary of a Cochrane Collaboration systematic review. J Cachexia Sarcopenia Muscle. 2015;6(3):208-11.

40. Akbulut G. New perspective for nutritional support of cancer patients: enteral/parenteral nutrition. Exp Ther Med. 2011:2(4):675-84.

41. Stojcev $Z$ et al. The role of dietary nutrition in stomach cancer. Contemp Oncol (Pozn). 2013;17(4):343-5.

42. MacDonald N. Cancer cachexia and targeting chronic inflammation: a unified approach to cancer treatment and palliative/supportive care. J Support Oncol. 2007;5(4):157-62.

43. Prado BL, Qian Y. Anti-cytokines in the treatment of cancer cachexia. Ann Palliat Med. 2019;8(1):67-79.

44. Burn J et al. Cancer prevention with aspirin in hereditary colorectal cancer (Lynch syndrome), 10-year followup and registry-based 20-year data in the CAPP2 study: a double-blind, randomised, placebo-controlled trial. Lancet. 2020;395(10240):1855-63.

45. Lin JL et al. Relationship between aspirin use of esophageal, gastric and colorectal cancer patient survival: a meta-analysis. BMC Cancer. 2020;20(1):638.

46. Persson C, Glimelius B. The relevance of weight loss for survival and quality of life in patients with advanced gastrointestinal cancer treated with palliative chemotherapy. Anticancer Res. 2002;22(6B):3661-8.

47. Dev $\mathrm{R}$ et al. The evolving approach to management of cancer cachexia. Oncology (Williston Park). 2017:31(1):23-32

48. Tisdale MJ. Clinical anticachexia treatments. Nutr Clin Pract. 2006:21(2):168-74.

49. Ruiz Garcia $\vee$ et al. Megestrol acetate for treatment of anorexia-cachexia syndrome. Cochrane Database Syst Rev. 2013;2013(3):CD004310.

50. Kouchaki B et al. Randomized double-blind clinical trial of combined treatment with megestrol acetate plus celecoxib versus megestrol acetate alone in cachexiaanorexia syndrome induced by GI cancers. Support Care Cancer. 2018;26(7):2479-89.

51. Mantovani $\mathrm{G}$ et al. Phase II nonrandomized study of the efficacy and safety of COX-2 inhibitor celecoxib on patients with cancer cachexia. J Mol Med (Berl). 2010;88(1):85-92.

52. Shirai $Y$ et al. Fish oil-enriched nutrition combined with systemic chemotherapy for gastrointestinal cancer patients with cancer cachexia. Sci Rep. 2017;7(1):4826.

53. Reid J et al. Thalidomide for managing cancer cachexia. Cochrane Database Syst Rev. 2012;2012(4):CD008664.

54. Khatib MN et al. Ghrelin as a promising therapeutic option for cancer cachexia. Cell Physiol Biochem. 2018;48(5):2172-88.

55. Temel JS et al. Anamorelin in patients with non-small-cell lung cancer and cachexia (ROMANA 1 and ROMANA 2): results from two randomised, double-blind, Phase 3 trials. Lancet Oncol. 2016;17(4):519-31

56. Hamauchi S et al. A multicenter, open-label, single-arm study of anamorelin (ONO-7643) in advanced gastrointestinal cancer patients with cancer cachexia. Cancer 2019:125(23):4294-302.

57. Grundmann $\mathrm{O}$ et al. Augmentation of cancer cachexia components with targeted acupuncture in patients with gastrointestinal cancers: a randomized controlled pilot study. Integr Cancer Ther. 2019;18:1534735418823269.

58. Del Fabbro E. Combination therapy in cachexia. Ann Palliat Med. 2019;8(1):59-66.

59. Roeland EJ et al. Management of cancer cachexia: ASCO guideline. J Clin Oncol. 2020;38(21):2438 